\title{
La utilización de precordiales derechas en el test de ejercicio mejora la detección de enfermedad coronaria
}

Improved detection of coronary artery disease by exercise electrocardiography with the use of right precordial leads. Michaelides A0, Psomadaki ZD, Dilaveris PE, et al. N Engl J Med 1999:340;340-345.

\section{Objetivo}

Demostrar que la combinación de precordiales derechas e izquierdas en el electrocardiograma de esfuerzo mejora la detección de enfermedad coronaria.

\section{Diseño}

Estudio de corte transversal.

\section{Lugar}

Comunidad de Boston Este, EEUU.

\section{Pacientes}

Se evaluaron 245 pacientes consecutivos con síntomas anginosos ( 218 hombres y 27 mujeres); edad media $52 \pm 8$ años (rango $32-74$ años). Todos realizaron test de ejercicio con cinta (treadmill), estudio de esfuerzo y redistribución con Talio 201 y cinecoronariografía.

\section{Descripción de los tests y del test de referencia}

El test de ejercicio con treadmill se realizó con el protocolo de Bruce. En cada paciente el ECG se realizó con las 12 derivaciones standard y 3 derivaciones precordiales derechas ( V3R, V4R y V5R). Los resultados fueron analizados en forma separada.

El test con Talio 201 (Ta201)se realizó con la inyección de 2 mci de Talio 201 en el pico del esfuezo y 3-4 horas después se obtuvieron las imágenes de redistribución.

El test de referencia fue la cinecoronariografía, definiendo enfermedad coronaria significativa como una estenosis $\geq 70 \%$ en el territorio de la arteria descendente anterior, coronaria derecha y/o circunfleja; o estenosis $\geq 50 \%$ de tronco de coronaria izquierda.

En todos los casos, los resultados fueron interpretados por 2 observadores independientes que no tenían conocimiento de los datos de los otros tests.

\section{Resultados principales}

De los 245 pacientes, 34 (14\%) presentaron coronarias normales, 85 (35\%) enfermedad de 1 vaso, 84 (34\%) de 2 vasos y $42(17 \%)$ de 3 vasos.De los pacientes con enfermedad de 1 vaso , 35 (41\%) presentaron enfermedad de arteria descendente anterior (DA) , 28 (33\%) de arteria coronaria derecha (CD) y 22 (26\%) de arteria circunfleja (CX). La sensibilidad del test de ejercicio con 12 derivaciones, test de ejercicio con 12 derivaciones +3 derivaciones precordiales derechas (" nueva técnica") y Ta 201 fue de $52 \%, 89 \%$ y $87 \%$ para la detección de enfermedad de 1 vaso $; 71 \%, 94 \%, 96 \%$ para la detección de enfermedad de 2 vasos; $83 \%, 95 \%$ y $98 \%$ para la detección de 3 vasos y de $66 \%$, $92 \%$ y $93 \%$ para la detección de coronariopatía.

En comparación con el ECG de ejercicio de 12 derivaciones, esta "nueva técnica" incrementa la sensibilidad en la detección de enfermedad en el territorio de la DA de 77 a $91 \%(p=0.06)$, de la arteria CD de 25 a $89 \%$ ( p 0.0001) y de la arteria $C X$ de 45 a $86 \%$ ( $p=0.004)$. La especificidad de la "nueva técnica" y Ta 201 fue de 80 y $82 \%$ (p NS).

\section{Conclusiones}

La utilización de las derivaciones precordiales derechas con precordiales izquierdas durante el test de ejercicio mejora significativamente la sensibilidad del test en la detección de enfermedad coronaria.

\section{COMENTARIO}

Las alteraciones del ST-T inducidas por el ejercicio identifica enfermedad coronaria con una sensibilidad de $68 \pm 16 \%$ y una especificidad de $77 \pm 17 \%$.

Esto depende de la probabilidad previa de enfermedad coronaria (pre-test)* , del número de vasos comprometidos, de las alteraciones del ST-T basales y del sexo (en el sexo femenino la especificidad es menor). Los autores de este trabajo presentan una "nueva técnica" que consiste en la adición de 3 precordiales derechas aL ECG de 12 derivaciones convencional durante el test de ejercicio. Utilizando la coronariografía como test de referencia, la "nueva técnica" presenta mayor sensibilidad que el ECG convencional y una similar seguridad diagnóstica que el Ta 201.

Algunos puntos a considerar en el estudio : a) Dado que solo el $11 \%$ de los pacientes son de sexo femenino, las conclusiones podrían no ser válidas en las mujeres; b) Los resultados se expresan como sensibilidad y especificidad, pero no se incluyen los intervalos de confian$z^{*}$ Esta información es relevante para saber el grado de precisión del resultado y poder considerarlo desde el punto de vista clínico. c) A pesar de estos comentarios, el estudio es metodológicamente sólido. La "nueva técnica" fue comparada en forma ciega con un test de referencia adecuado y presentó resultados comparables con estudios más costosos como el Ta 201, favoreciendo además la mejoría de la sensibilidad diagnóstica en el territorio de la CD y CX.

Por lo tanto, considero que la adición de las precordiales derechas al ECG de esfuerzo convencional, resulta de gran valor en la detección de enfermedad coronaria.

\section{*Ver Glosario}

\section{Dr. Rodolfo Pizarro}

Servicio de Cardiología. Hospital Italiano de Buenos Aires.

\section{Referencias}

1-Gianrossi R, Detrano R, Mulvihill D, et al : Exercise induced ST depression in the diagnosis of coronary artery disease : a meta-analysis. Circulation 1989;80;87-98.

2-Krueger DW, Lesnesfsky EJ, Groves BM, et al:Right ventricular ischemia and proximal right coronary artery narrowing indicated by excercise ST- elevation in lead V4.Am J Cardio $1989 ; 63 ; 107-109$

3-Candell-Riera J, Figueras J, Valle V et al: Right ventricular infarction:relationships between ST segment elevation in V4R and henodynamics,5cintigraphic and echocardiographic findings in patients with acute inferior myocardial infarction.Am Heart J 1981;101;281-287.

4-Braat SH,Zwaan C, Brugada P, et al: Value of electrocardiogram in diagnosis right ventricular involvement in patients with an acute inferior wall myocardial infarction. Br Heart $\mathrm{J}$ $1983 ; 47 ; 368-372$

5-Zehender M, Kasper W, Kauder E, et al:Right ventricular infarction as an independent predictor of prognosis after acute inferior myocardial infarction.N Engl J Med 1993;328;981-988. 6-Wellens HJJ.Right ventricular infarction. N Engl J Med 1993;328;1036-1038. 